\title{
Nonextensive triangle equality and other properties of Tsallis relative-entropy minimization
}

\author{
Ambedkar Dukkipati, ${ }^{1}$ M. Narasimha Murty, ${ }^{* 2}$ \\ Shalabh Bhatnagar ${ }^{3}$ \\ Department of Computer Science and Automation, Indian Institute of Science, \\ Bangalore-560012, India.
}

\begin{abstract}
Kullback-Leibler relative-entropy has unique properties in cases involving distributions resulting from relative-entropy minimization. Tsallis relative-entropy is a one parameter generalization of Kullback-Leibler relative-entropy in the nonextensive thermostatistics. In this paper, we present the properties of Tsallis relative-entropy minimization and present some differences with the classical case. In the representation of such a minimum relative-entropy distribution, we highlight the use of the $q$-product, an operator that has been recently introduced to derive the mathematical structure behind the Tsallis statistics. One of our main results is generalization of triangle equality of relative-entropy minimization to the nonextensive case.
\end{abstract}

Key words: ME methods, Tsallis entropy, triangle equality

PACS: 02.50.-r, 05.20.-y, 02.70.Rr

\section{Introduction}

Maximum and minimum entropy methods, known as ME methods, originally coming from physics, have been promoted to a general principle of inference primarily by the works of Jaynes [1] and later by Kullback [2]. Jaynes maximum entropy principle involves maximizing Shannon entropy $[3,4]$ while

\footnotetext{
* corresponding author

1 ambedkar@csa.iisc.ernet.in

2 mnm@csa.iisc.ernet.in (Tel:+91-80-22932779)

3 shalabh@csa.iisc.ernet.in
} 
Kullback minimum entropy principle involves minimizing Kullback-Leibler relative-entropy [2]. Logarithmic form of information measure is common for all these entropies.

On the other hand, however, Tsallis in [5] proposed a non-logarithmic form of entropy (termed as nonextensive entropy or Tsallis entropy) which is considered as a useful measure in describing thermostatistical properties of a certain class of physical systems that entail long-range interactions, long-term memories and multi-fractal structures. The thermostatistical formalism based on Tsallis entropy is termed as generalized or nonextensive thermostatistics, since Tsallis entropy is a one-parameter generalization of Shannon entropy and does not satisfy additive property involving independent probability distributions but satisfies the so-called pseudo additivity or nonextensive additivity (see (9)). These generalized statistics have been applied not only to physical systems but also to various problems in optimization (generalized simulated annealing [6]), statistical inference [7], machine learning [8] etc. Recently Shannon-Khinchin axioms have been generalized to nonextensive systems [9].

On similar lines, generalization of relative-entropy, called Tsallis relative-entropy, has been proposed in [7]. In this paper, we study Tsallis relative-entropy minimization and its differences with the classical case. We generalize the triangle equality (see (6)) of Kullback-Leibler relative-entropy minimization, which qualifies relative-entropy minimization as an optimal inference procedure with respect to relative-entropy as an information measure, to Tsallis relative-entropy case.

We present the necessary background in $\S 2$, where we discuss properties of relative-entropy minimization in the classical case, and we give the basic definitions related to Tsallis entropy. In $\S 3$ we present the relative-entropy minimization in non-extensive framework and discuss its differences with the classical case. Finally, triangle equality for Tsallis relative-entropy minimization is derived in $\S 4$. A brief discussion of Tsallis relative-entropy minimization in the case of "normalized $q$-expected" values is presented in $\S 5$.

\section{Background}

\subsection{Relative-entropy minimization: In classical case}

Minimizing the Kullback-Leibler relative-entropy (or I-divergence or crossentropy) with respect to a set of moment constraints finds its importance in the celebrated Kullback's minimum relative-entropy principle [2]. This principle is a general method of inference about an unknown probability distribution 
when there exists a prior estimate of the distribution and new information in the form of constraints on expected values [10]. Formally, we can state the minimum relative-entropy principle as: given a prior distribution $r$, of all the probability distributions that satisfy the given moment constraints, one should choose the posterior $p$ with the least relative-entropy

$$
I_{1}(p \| r)=\int d x p(x) \ln \frac{p(x)}{r(x)}
$$

provided that the integral above exists ${ }^{4}$. The prior distribution $r$ can be a reference distribution (uniform, Gaussian, Lorentzian or Boltzmann etc.) or a prior estimate of $p$.

The principle of Jaynes maximum entropy is a special case of minimization of relative-entropy under appropriate conditions [13]. In particular, minimizing relative-entropy is equivalent to maximizing Shannon entropy when the prior is a uniform distribution. Relative-entropy minimization has been applied primarily to statistics [2], and also to statistical mechanics [14], pattern recognition [15], spectral analysis [16], speech coding [17]. For a list of references on applications of relative-entropy minimization see $[13,12]$.

Properties of relative-entropy minimization have been studied and presented extensively in [10]. Here we briefly mention a few. Given a prior distribution $r$ with a finite set of moment constraints of the form

$$
\int d x u_{m}(x) p(x)=\left\langle u_{m}\right\rangle, m=1, \ldots M
$$

along with the normalizing constraint $\int p(x) d x=1$ (from now on we assume that any set of constraints on probability distributions implicitly includes this constraint), the minimum relative-entropy distribution is of the form

$$
p(x)=\frac{r(x) e^{-\sum_{m=1}^{M} \beta_{m} u_{m}(x)}}{\widehat{Z_{1}}},
$$

where

$$
\widehat{Z_{1}}=\int d x r(x) e^{-\sum_{m=1}^{M} \beta_{m} u_{m}(x)}
$$

4 In measure theoretic terms, the integral exists if the measure induced by $p$ is absolutely continuous with respect to that induced by $r$, otherwise $I_{1}(p \| r)=\infty$ [11]. In this work we do not aim at mathematical rigor of the measure theoretic information theory. In particular, we assume that all quantities of interest exist for all distributions considered. Note that the measure theoretic definitions of these quantities relies strongly on the Lebesgue-Radon-Nikodym Theorem [12]. 
is the partition function, $\beta_{m}, m=1, \ldots, M$ are the corresponding Lagrange multipliers, $u_{m}, m=1, \ldots M$ are some functions of the underlying random variable whose expectation values $\left\langle u_{m}\right\rangle, m=1, \ldots M$ are (assumedly) a priori known. When the prior is a uniform distribution with a compact support of $W$ ( $W$ possible configurations in discrete case), the minimum relative-entropy distribution turns out to be

$$
p(x)=\frac{e^{-\sum_{m=1}^{M} \beta_{m} u_{m}(x)}}{\int d x e^{-\sum_{m=1}^{M} \beta_{m} u_{m}(x)}},
$$

which is in fact a maximum entropy distribution (Boltzmann distribution) of Shannon entropy with respect to the constraints (2).

Many properties of relative-entropy minimization just reflect well-known properties of relative-entropy but there are surprising differences as well [10]. For example, relative-entropy does not generally satisfy a triangle relation involving three arbitrary probability distributions. But in certain important cases involving distributions that result from relative-entropy minimization, relativeentropy does satisfy triangle equality.

The statement of triangle equality can be formulated as follows. Let $r$ be the prior distribution, $p$ be the probability distribution that minimizes the relative-entropy subject to set of constraints (2) and $l$ be any other distribution satisfying the same constraints, then we have the triangle equality [10]:

$$
I_{1}(l \| r)=I_{1}(l \| p)+I_{1}(p \| r)
$$

This triangle equality is important for application in which relative-entropy minimization is used for purposes of pattern classification and cluster analy$\operatorname{sis}[15]$.

\subsection{Nonextensive framework}

Tsallis entropy, which was introduced by Tsallis [5] is given by

$$
S_{q}(p)=-\int d x p(x) \frac{p(x)^{q-1}-1}{q-1}
$$

where $q \in \mathbb{R}$ is called non-extensive index ( $q$ is positive in order to ensure the concavity of $S_{q}$ ). Tsallis entropy is a one-parameter generalization of Shannon 
entropy in the sense that

$$
\lim _{q \rightarrow 1} S_{q}(p)=-\int d x p(x) \ln p(x)=S_{1}(p)
$$

The entropic index $q$ characterizes the degree of nonextensivity reflected in the pseudo-additivity property

$$
S_{q}(A+B)=S_{q}(A)+S_{q}(B)+(1-q) S_{q}(A) S_{q}(B)
$$

where $A$ and $B$ are two independent systems in the sense that the probability distribution of $A+B$ factorizes into those of $A$ and $B$.

Maximizing the Tsallis entropy $S_{q}$ with respect to the constraints

$$
\int d x u_{m}(x) p(x)^{q}=\left\langle u_{m}\right\rangle_{q}, \quad m=1, \ldots M
$$

the generalized equilibrium probability distribution is found to be [18],

$$
p(x)=\frac{\left[1-(1-q) \sum_{m=1}^{M} \beta_{m} u_{m}(x)\right]^{\frac{1}{1-q}}}{Z_{q}}
$$

where

$$
Z_{q}=\int d x\left[1-(1-q) \sum_{m=1}^{M} \beta_{m} u_{m}(x)\right]^{\frac{1}{1-q}}
$$

is the partition function, and $\beta_{m}, m=1, \ldots M$ are the corresponding Lagrange multipliers ${ }^{5}$ and $\left\langle u_{m}\right\rangle_{q}$ is a known $q$-expectation of $u_{m}$ [18]. This distribution is called generalized maximum entropy distribution or simply Tsallis distribution [19]. The limit $q \rightarrow 1$ in (11), recovers the maximum entropy distribution in the classical case.

5 To avoid proliferation of symbols we use same notation for the minimum or maximum entropy distributions and Lagrange multipliers in the various cases; the correspondence should be clear from the context. 
Now, the definition of Kullback-Leibler relative-entropy $I_{1}(1)$ and the generalized entropic form $S_{q}(7)$ naturally lead to the generalization [7]

$$
I_{q}(p \| r)=\int d x p(x) \frac{\left[\frac{p(x)}{r(x)}\right]^{q-1}-1}{q-1},
$$

which is called as Tsallis relative-entropy. The limit $q \rightarrow 1$ recovers the relative-entropy in the classical case. Also one can verify that (see [7] )

$$
\begin{aligned}
I_{q}(p \| r) \geq 0 & \text { if } q>0 \\
& =0 \text { if } q=0 \\
& \leq 0 \text { if } q<0
\end{aligned}
$$

For $q \neq 0$, the equalities hold if and only if $p=r$ almost everywhere. Further, for $q>0, I_{q}(p \| r)$ is a convex function of $p$ and $r$, and for $q<0$ it is concave [20]. Like Tsallis entropy, Tsallis relative-entropy satisfies the pseudoadditivity of the form [21]

$$
\begin{aligned}
I_{q}\left(A_{1}+A_{2} \| B_{1}+B_{2}\right)=I_{q}\left(A_{1} \| B_{1}\right) & +I_{q}\left(A_{2} \| B_{2}\right) \\
& +(q-1) I_{q}\left(A_{1} \| B_{1}\right) I_{q}\left(A_{2} \| B_{2}\right),
\end{aligned}
$$

where $A_{1}, A_{2}$ and $B_{1}, B_{2}$ are the independent pairs. The limit $q \rightarrow 1$ in (15) retrieves

$$
I_{1}\left(A_{1}+A_{2} \| B_{1}+B_{2}\right)=I_{1}\left(A_{1} \| B_{1}\right)+I_{1}\left(A_{2} \| B_{2}\right)
$$

the additivity property of Kullback-Leibler relative-entropy.

Further properties of Tsallis relative-entropy have been discussed in [7,20,21]. Characterization of Tsallis relative-entropy, by generalizing Hobson's uniqueness theorem [22] of relative-entropy, is presented in [23]. 


\section{Tsallis relative-entropy minimization}

\subsection{Generalized minimum relative-entropy distribution}

To minimize Tsallis relative-entropy with respect to the set of constraints (10) the concomitant variational principle can be written as

$$
\delta\left\{I_{q}(p \| r)+\lambda\left(\int d x p(x)-1\right)+\sum_{m=1}^{M} \beta_{m}\left(\int d x p(x)^{q} u_{m}(x)-\left\langle u_{m}\right\rangle_{q}\right)\right\}=0
$$

where $\lambda$ and $\beta_{m}, m=1, \ldots M$ are Lagrange multipliers. This gives us minimum Tsallis relative-entropy distribution as [20]

$$
p(x)=\frac{\left[r(x)^{1-q}-(1-q) \sum_{m=1}^{M} \beta_{m} u_{m}(x)\right]^{\frac{1}{1-q}}}{\widehat{Z}_{q}},
$$

where values of $\beta_{m}, m=1 \ldots M$ are determined by the constraints (10) and $\widehat{Z_{q}}$, the partition function, is given by

$$
\widehat{Z_{q}}=\int d x\left[r(x)^{1-q}-(1-q) \sum_{m=1}^{M} \beta_{m} u_{m}(x)\right]^{\frac{1}{1-q}} .
$$

A note on the constraint (10). This constraint had been used for some time [24], but because of problems in justifying it on physical grounds the constraint

$$
\frac{\int d x p(x)^{q} u_{m}(x)}{\int d x p(x)^{q}}=\left\langle\left\langle u_{m}\right\rangle\right\rangle_{q}
$$

has been introduced in [18]. $\left\langle\left\langle u_{m}\right\rangle\right\rangle_{q}$ is called normalized q-expectation value of $u_{m}$. We discuss Tsallis relative-entropy minimization and its properties with respect to the constraint (19), briefly, in $\S 5$.

\section{2 q-product representation of generalized minimum relative-entropy distri- bution}

The mathematical basis for Tsallis statistics comes from the q-deformed expressions for the logarithm $(q$-logarithm $)$ and the exponential function ( $q$ - 
exponential) which were first proposed in [25], in the context of nonextensive thermostatistics. The $q$-logarithm is defined as

$$
\ln _{q} x=\frac{x^{1-q}-1}{1-q}(x>0, q \in \mathbb{R})
$$

and the $q$-exponential is defined as

$$
e_{q}^{x}= \begin{cases}{[1+(1-q) x]^{\frac{1}{1-q}}} & \text { if } 1+(1-q) x \geq 0 \\ 0 & \text { otherwise. }\end{cases}
$$

These two functions are related by

$$
e_{q}^{\ln _{q} x}=x
$$

Properties of these q-deformed functions are studied in [26]. In this framework a new multiplication operation, called $q$-product

$$
x \otimes_{q} y \equiv \begin{cases}\left(x^{1-q}+y^{1-q}-1\right)^{\frac{1}{1-q}} & \text { if } x, y>0 \text { and } x^{1-q}+y^{1-q}-1>0 \\ 0 & \text { otherwise }\end{cases}
$$

is first introduced in [27] and explicitly defined in [28] for satisfying the following equations:

$$
\begin{aligned}
\ln _{q}\left(x \otimes_{q} y\right) & =\ln _{q} x+\ln _{q} y, \\
e_{q}^{x} \otimes_{q} e_{q}^{y} & =e_{q}^{x+y} .
\end{aligned}
$$

The $q$-product recovers the usual product in the limit $q \rightarrow 1$ i.e., $\lim _{q \rightarrow 1}\left(x \otimes_{q}\right.$ $y)=x y$. The fundamental properties of the $q$-product $\otimes_{q}$ are almost the same as the usual product, and in general

$$
a\left(x \otimes_{q} y\right) \neq a x \otimes_{q} y \quad(a, x, y \in \mathbb{R}) .
$$

Further properties of the $q$-product can be found in [27,28]. Also, $q$-product has been used in various applications of Tsallis statistics [29].

Previously, generalized entropies and maximum entropy distributions have been represented in terms of $q$-logarithm and $q$-exponential. We list some of 
them which we are going to use later in this paper. Tsallis entropy (7) can be represented as

$$
S_{q}(p)=-\int d x p(x)^{q} \ln _{q} p(x)
$$

and Tsallis relative-entropy (13) as

$$
I_{q}(p \| r)=-\int d x p(x) \ln _{q} \frac{r(x)}{p(x)}
$$

One can represent Tsallis distribution (11) in terms of $q$-exponential as

$$
p(x)=\frac{e_{q}^{-\sum_{m=1}^{M} \beta_{m} u_{m}(x)}}{Z_{q}},
$$

since $p(x)=0$ whenever $\left[1-(1-q) \sum_{m=1}^{M} \beta_{m} u_{m}(x)\right]<0$ which is Tsallis cut-off condition [5] assumed implicitly.

Note that generalized relative-entropy distribution (17), is not of the form of (3) even if we replace the exponential with the q-exponential. But one can verify the non-trivial fact that (17) can be expressed in a similar form as in the classical case by invoking q-product as,

$$
p(x)=\frac{r(x) \otimes_{q} e_{q}^{-\sum_{m=1}^{M} \beta_{m} u_{m}(x)}}{\widehat{Z}_{q}}
$$

where

$$
\widehat{Z_{q}}=\int d x r(x) \otimes_{q} e_{q}^{-\sum_{m=1}^{M} \beta_{m} u_{m}(x)}
$$

One can see from the later parts of this paper, this representation is useful in deriving properties of Tsallis relative-entropy minimization.

It is important to note that the distribution in (17) could be a (local/global) minimum only if $q>0$ and Tsallis cut-off condition is extended to the relativeentropy case i.e., $p(x)=0$ whenever $\left[r(x)^{1-q}-(1-q) \sum_{m=1}^{M} \beta_{m} u_{m}(x)\right]<0$. 


\subsection{Properties}

As we mentioned earlier, in the classical case i.e., when $q=1$, relative-entropy minimization with uniform distribution as a prior is equivalent to entropy maximization. But, in the case of nonextensive framework, this is not true. Let $r$ be the uniform distribution with compact support $W$ over $E \subset \mathbb{R}$. Then, by (17) one can verify that probability distribution which minimizes Tsallis relative-entropy is

$$
p(x)=\frac{\left[\frac{1}{W^{1-q}}-(1-q) \sum_{m=1}^{M} \beta_{m} u_{m}(x)\right]^{\frac{1}{1-q}}}{\int_{E} d x\left[\frac{1}{W^{1-q}}-(1-q) \sum_{m=1}^{M} \beta_{m} u_{m}(x)\right]^{\frac{1}{1-q}}},
$$

which can be written as (by (39) and (42))

$$
p(x)=\frac{e_{q}^{-W^{q-1} \ln _{q} W-\sum_{m=1}^{M} \beta_{m} u_{m}(x)}}{\int_{E} d x e_{q}^{-W^{q-1} \ln _{q} W-\sum_{m=1}^{M} \beta_{m} u_{m}(x)}}
$$

or (by the definition of $q$-exponential (21))

$$
p(x)=\frac{e_{q}^{-W^{1-q} \sum_{m=1}^{M} \beta_{m} u_{m}(x)}}{\int_{E} d x e_{q}^{-W^{1-q} \sum_{m=1}^{M} \beta_{m} u_{m}(x)}} .
$$

By comparing (30) or (31) with Tsallis maximum entropy distribution (28) one can conclude (formally one can verify this by thermodynamical equations of Tsallis entropy [5]) that minimizing relative-entropy is not equivalent ${ }^{6}$ to maximizing entropy when the prior is uniform distribution. The key observation here is $W$ appeares in (31) unlike (28).

$\overline{6}$ For fixed $q$-expected values $\left\langle u_{m}\right\rangle_{q}$, the two distributions, (31) and (28) are equal, but the values of corresponding Lagrange multipliers are different when $q \neq 1$ (while in the classical case they remain same). Further, (31) offers the relation between the Lagrange parameters in these two cases. Let $\beta_{m}^{(S)}, m=1, \ldots M$ corresponds to the Lagrange parameters corresponds to the generalized maximum entropy distribution while $\beta_{m}^{(I)}, m=1, \ldots M$ corresponds to generalized minimum relative-entropy distribution with uniform prior. Then, we have relation $\beta_{m}^{(S)}=W^{1-q} \beta_{m}^{(I)}, m=1, \ldots M$. 
Also minimum Tsallis relative-entropy satisfies [20]:

$$
I_{q}(p \| r)=-\ln _{q} \widehat{Z_{q}}-\sum_{m=1}^{M} \beta_{m}\left\langle u_{m}\right\rangle_{q}
$$

to prove which, one can escape lengthy algebraic manipulations by using $q$ deformed representations of various formulae.

The thermodynamic equations for the minimum Tsallis relative-entropy are

$$
\begin{gathered}
\frac{\partial}{\partial \beta_{m}} \ln _{q} \widehat{Z_{q}}=-\left\langle u_{m}\right\rangle_{q}, \quad m=1, \ldots M \\
\frac{\partial I_{q}}{\partial\left\langle u_{m}\right\rangle_{q}}=-\beta_{m}, \quad m=1, \ldots M
\end{gathered}
$$

which generalize thermodynamic equations in the classical case. One should note that these thermodynamic equations were proved to hold true for, essentially, any entropic measure [30,31].

\section{Nonextensive triangle equality}

Before we derive equivalent of triangle equality in the nonextensive thermostatistics we shall discuss the significance of triangle equality of KullbackLeibler relative-entropy minimization. Significance of triangle equality comes in the following scenario. Let $r$ be the prior estimate of the unknown probability distribution $l$ about which information in the form of expected value constraints

$$
\int d x u_{m}(x) l(x)=\left\langle u_{m}\right\rangle, \quad m=1, \ldots M
$$

is available for fixed functions $u_{m}, m=1, \ldots M$. The problem is to choose a posterior estimate $p$ that is in some sense the best estimate of $l$ given the available information i.e., prior and the information in the form of expected values. The principle of minimum entropy provides a general solution to this inference problem and provides us the estimate (3). Further, from triangle equality (6), the minimum relative-entropy posterior estimate of $l$ is not only logically consistent, but also closer to $l$, in the relative-entropy sense, than is the prior $r$. Moreover, the difference $I_{1}(l \| r)-I_{1}(l \| p)$ is exactly the relativeentropy $I_{1}(p \| r)$ between the posterior and the prior. Hence $I_{1}(p \| r)$ can be interpreted as the amount of information provided by the constraints that is

not inherent in $r$. 
Additional justification to use minimum relative-entropy estimate of $p$ with respect to constraints (2) is provided by the following expected value matching property [10]. For fixed functions $u_{m}, m=1, \ldots M$, let the actual unknown distribution $l$ satisfy

$$
\int d x u_{m}(x) l(x)=\left\langle w_{m}\right\rangle, \quad m=1, \ldots M
$$

Now, as $\left\langle u_{m}\right\rangle, m=1, \ldots M$ vary, $I_{q}(l \| p)$ has the minimum value when

$$
\left\langle u_{m}\right\rangle=\left\langle w_{m}\right\rangle, \quad m=1, \ldots M
$$

For the proof of expected value matching property see [10]. This property states that for a distribution $p$ of the form $(3), I_{1}(l \| p)$ is the smallest when the expected values of $p$ match those of $l$. In particular, $p$ is not only the distribution that minimizes $I_{1}(p \| r)$ but also is the distribution of the form (3) that minimizes $I_{1}(l \| p)$. This property is a generalization of a property of orthogonal polynomials [32] which in the case of speech analysis [17] is called the "correlation matching property".

From the above discussion, it is clear that to derive a triangle equality of Tsallis relative-entropy minimization, one should first deduce the equivalent of expectation matching property in the nonextensive case. Let $l$ be the actual unknown distribution which satisfies

$$
\int d x u_{m}(x) l(x)^{q}=\left\langle w_{m}\right\rangle_{q}, \quad m=1, \ldots M
$$

$r$ be the prior estimate of $l$ and $p$ be the posterior which satisfies constraints (10). That is, we would like to find the values of $\left\langle u_{m}\right\rangle_{q}$ for which $I_{q}(l, p)$ is minimum. We write the following useful relations before we proceed to the derivation.

We can write generalized minimum relative-entropy distribution (29) as

$$
p(x)=\frac{e_{q}^{\ln _{q} r(x)} \otimes_{q} e_{q}^{-\sum_{m=1}^{M} \beta_{m} u_{m}(x)}}{\widehat{Z}_{q}}=\frac{e_{q}^{-\sum_{m=1}^{M} \beta_{m} u_{m}(x)+\ln _{q} r(x)}}{\widehat{Z}_{q}}
$$

by (22) and (25). Further by using

$$
\ln _{q}(x y)=\ln _{q} x+\ln _{q} y+(1-q) \ln _{q} x \ln _{q} y
$$


we get the relation

$$
\ln _{q} p(x)+\ln _{q} \widehat{Z_{q}}+(1-q) \ln _{q} p(x) \ln _{q} \widehat{Z_{q}}=-\sum_{m=1}^{M} \beta_{m} u_{m}(x)+\ln _{q} r(x)
$$

By the property of $q$-logarithm [33]

$$
\ln _{q}\left(\frac{x}{y}\right)=y^{q-1}\left(\ln _{q} x-\ln _{q} y\right)
$$

and by (26), (27) one can verify that

$$
I_{q}(p \| r)=-\int d x p(x)^{q} \ln _{q} r(x)-S_{q}(p) .
$$

To proceed with the derivation, consider

$$
I_{q}(l \| p)=-\int d x l(x) \ln _{q} \frac{p(x)}{l(x)} .
$$

By (42) we have

$$
\begin{aligned}
I_{q}(l \| p) & =-\int d x l(x)^{q}\left[\ln _{q} p(x)-\ln _{q} l(x)\right] \\
& =I_{q}(l \| r)-\int d x l(x)^{q}\left[\ln _{q} p(x)-\ln _{q} r(x)\right] .
\end{aligned}
$$

From (41), we get

$$
\begin{aligned}
I_{q}(l \| p)=I_{q}(l \| r)+\int d x l(x)^{q} & \left(\sum_{m=1}^{M} \beta_{m} u_{m}(x)\right)+\ln _{q} \widehat{Z_{q}} \int d x l(x)^{q} \\
& +(1-q) \ln _{q} \widehat{Z_{q}} \int d x l(x)^{q} \ln _{q} p(x)
\end{aligned}
$$

By using (38) and (43),

$$
\begin{aligned}
I_{q}(l \| p)=I_{q}(l \| r)+\sum_{m=1}^{M} \beta_{m}\left\langle w_{m}\right\rangle_{q} & +\ln _{q} \widehat{Z_{q}} \int d x l(x)^{q} \\
& +(1-q) \ln _{q} \widehat{Z}_{q}\left[-I_{q}(l \| p)-S_{q}(l)\right]
\end{aligned}
$$


and by (7) we have

$$
I_{q}(l \| p)=I_{q}(l \| r)+\sum_{m=1}^{M} \beta_{m}\left\langle w_{m}\right\rangle_{q}+\ln _{q} \widehat{Z_{q}}-(1-q) \ln _{q} \widehat{Z_{q}} I_{q}(l \| p) .
$$

Since the multipliers $\beta_{m}, \quad m=1, \ldots M$ are functions of the expected values $\left\langle u_{m}\right\rangle_{q}$, variations in the expected values are equivalent to variations in the multipliers. Hence to find the minimum of $I_{q}(l, p)$, we solve

$$
\frac{\partial}{\partial \beta_{m}} I_{q}(l \| p)=0
$$

which gives us

$$
\left\langle u_{m}\right\rangle_{q}=\frac{\left\langle w_{m}\right\rangle_{q}}{1-(1-q) I_{q}(l \| p)}, \quad m=1, \ldots M
$$

In the limit $q \rightarrow 1$ the above equation gives $\left\langle u_{m}\right\rangle_{1}=\left\langle w_{m}\right\rangle_{1}$ which is the expectation matching property in the classical case.

Now, to derive the triangle equality for Tsallis relative-entropy minimization,

we substitute the expression for $\left\langle w_{m}\right\rangle_{q}$, which is given by (48), in (47). And after some algebra one can arrive at

$$
I_{q}(l \| r)=I_{q}(l \| p)+I_{q}(p \| r)+(q-1) I_{q}(l \| p) I_{q}(p \| r) .
$$

The limit $q \rightarrow 1$ in (49) gives the triangle equality in the classical case (6). The two important cases which arise out of (49) are,

$$
\begin{array}{ll}
I_{q}(l \| r) \leq I_{q}(l \| p)+I_{q}(p \| r) & \text { when } 0<q \leq 1 \\
I_{q}(l \| r) \geq I_{q}(l \| p)+I_{q}(p \| r) & \text { when } 1<q .
\end{array}
$$

We call (49) as nonextensive triangle equality, whose pseudo additivity is consistant with the pseudo additivity of Tsallis relative-entropy (compare (15) and (16)), and hence is a natural generalization of triangle equality in the classical case.

\section{In the case of 'normalized q-expectations'}

In this Section we discuss Tsallis relative-entropy minimization with respect to the constraints in the form of normalized $q$-expectations (19). For a complete 
discussion on choice of constraints (10) and (19) for Tsallis entropy maximization see $[18,34]$.

The variational principle for Tsallis relative-entropy minimization with respect to (19) can be written as

$$
\delta\left\{I_{q}(p \| r)+\lambda\left(\int d x p(x)-1\right)+\sum_{m=1}^{M} \beta_{m}\left(\frac{\int d x p(x)^{q} u(x)}{\int d x p(x)^{q}}-\left\langle\left\langle u_{m}\right\rangle\right\rangle_{q}\right)\right\}=0
$$

where $\lambda$ and $\beta_{m}, m=1, \ldots M$ are Lagrange multipliers. This gives generalized minimum relative-entropy distribution as

$$
p(x)=\frac{\left[r(x)^{1-q}-(1-q) \frac{\sum_{m=1}^{M} \beta_{m}\left(u_{m}(x)-\left\langle\left\langle u_{m}\right\rangle\right\rangle_{q}\right)}{\int d x p(x)^{q}}\right]^{\frac{1}{1-q}}}{\widehat{\widehat{Z_{q}}}},
$$

where

$$
\widehat{\overline{Z_{q}}}=\int d x\left[r(x)^{1-q}-(1-q) \frac{\sum_{m=1}^{M} \beta_{m}\left(u_{m}(x)-\left\langle\left\langle u_{m}\right\rangle\right\rangle_{q}\right)}{\int d x p(x)^{q}}\right]^{\frac{1}{1-q}} .
$$

$q \rightarrow 1$ in (52) retrieves the minimum relative-entropy distribution in the classical case.

This can be expressed as

$$
p(x)=\frac{r(x) \otimes_{q} e_{q}^{-\sum_{m=1}^{M} \beta_{m}^{\prime}\left(u_{m}(x)-\left\langle\left\langle u_{m}\right\rangle\right\rangle_{q}\right)}}{\widehat{\bar{Z}_{q}}}
$$

where $^{7}$

$$
\beta_{m}^{\prime}=\frac{\beta_{m}}{\int d x p(x)^{q}}, \quad m=1, \ldots M
$$

Minimum Tsallis relative-entropy in this case satisfies

$$
I_{q}(p \| r)=-\ln _{q} \widehat{\overline{Z_{q}}}
$$

while corresponding thermodynamical equations can be written as

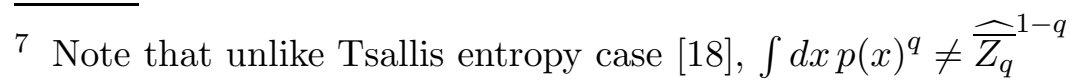




$$
\begin{gathered}
\frac{\partial}{\partial \beta_{m}} \ln _{q} \widehat{Z}_{q}=-\left\langle\left\langle u_{m}\right\rangle\right\rangle_{q}, \quad m=1, \ldots M, \\
\frac{\partial I_{q}}{\partial\left\langle\left\langle u_{m}\right\rangle\right\rangle_{q}}=-\beta_{m}, \quad m=1, \ldots M,
\end{gathered}
$$

where

$$
\ln _{q} \widehat{Z_{q}}=\ln _{q} \widehat{\widehat{Z_{q}}}-\sum_{m=1}^{M} \beta_{m}\left\langle\left\langle u_{m}\right\rangle\right\rangle_{q} .
$$

Now using above relations one can prove that, in this case too, Tsallis relativeentropy satisfies non-extensive triangle equality with modified conditions from the case of $q$-expectation values. We state it formally as follows. Let $r$ be the prior estimate of the unknown distribution $l$ which satisfies

$$
\frac{\int d x u_{m}(x) l(x)^{q}}{\int d x l(x)^{q}}=\left\langle\left\langle w_{m}\right\rangle\right\rangle_{q}, \quad m=1, \ldots M,
$$

where $\left\langle\left\langle w_{m}\right\rangle\right\rangle_{q}, m=1, \ldots M$ are known normalized $q$-expected values of $l$. Let $p$ be the posterior which satisfies constraints (19). Then, similar to the calculations in $\S 4$ one can prove that Tsallis relative entropy satisfies the nonextensive triangle equality (49), provided

$$
\left\langle\left\langle u_{m}\right\rangle\right\rangle_{q}=\left\langle\left\langle w_{m}\right\rangle\right\rangle_{q} m=1, \ldots M,
$$

but the minimum of $I_{q}(l \| p)$ is not guaranteed. Note that this condition is same as expectation value matching property in the classical case (see (37).

The detailed study of Tsallis relative-entropy minimization in this case of normalized $q$-expected values and the computation of corresponding minimum relative-entropy distribution (where one has to address the self-referential nature of the probabilities $p(x)$ in (52)) based on Tsallis et. al [18], Martínez et. al [34] formalisms for Tsallis entropy maximization is under study.

\section{Conclusions}

Tsallis relative-entropy minimization has been studied and some significant differences with the classical case are presented. Generalized relative-entropy minimization has been shown to satisfy an appropriate generalized version of triangle equality for the classical case. This is yet another remarkable and consistant generalization shown by Tsalls statistics. Considering the various 
fields to which Tsallis generalized statistics has been applied, studies of applications of Tsallis relative-entropy minimization for various inference problems are welcome.

\section{Acknowledgments}

The authors wish to thank anonymous refere for the comments. The authors thank Prof. C. Tsallis for encouraging them to publish the present material upon going through the contents.

\section{References}

[1] E. T. Jaynes, Papers on Probability, Statistics and Statistical Physics, D. Reidel publishing Co., Dordrecth, Holland, 1983.

[2] S. Kullback, Information Theory and Statistics, Wiley, New York, 1959.

[3] W. M. Elsasser, On quantum measurements and the role of the uncertainty relations in statistical mechanics, Physical Review 52 (1937) 987-999.

[4] E. T. Jaynes, Information theory and statistical mechanics, Physical Review 106 (4) (1957) 620-630.

[5] C. Tsallis, Possible generalization of Boltzmann Gibbs statistics, J. Stat. Phys. 52 (1988) 479.

[6] C. Tsallis, D. A. Stariolo, Generalized simulated annealing, Physica A 233 (1996) 345-406.

[7] C. Tsallis, Generalized entropy-based criterion for consistent testing, Physical Review E 58 (1998) 1442-1445.

[8] A. D. Anastasiadis, G. D. Magoulas, Nonextensive statistical mechanics for hybrid learning of neural networks, Physica A 344 (2004) 372-382.

[9] H. Suyari, Generalization of Shannon-Khinchin axioms to nonextensive systems and the uniqueness theorem for the nonextensive entropy, IEEE Transactions on Information Theory 50 (8) (2004) 1783-1787.

[10] J. E. Shore, Properties of cross-entropy minimization, IEEE Transactions on Information Theory IT-27 (4) (1981) 472-482.

[11] I. Csiszár, I-divergence of probability distributions and minimization problems, Ann. Prob. 3 (1) (1975) 146-158. 
[12] A. S. Cherney, V. P. Maslov, On minimization and maximization of entropy in various disciplines, SIAM journal of Theory of Probability and Its Applications 48 (3) (2004) 447-464.

[13] J. E. Shore, R. W. Johnson, Axiomatic derivation of the principle of maximum entropy and the principle of minimum cross-entropy, IEEE Transactions on Information Theory IT-26 (1) (1980) 26-37.

[14] A. Hobson, Concepts in Statistical Mechanics, Gordon and Breach, New York, 1971.

[15] J. E. Shore, R. M. Gray, Minimum cross-entropy pattern classification and cluster analysis, IEEE Transactions on Pattern Analysis and Machine Intelligence 4 (1) (1982) 11-18.

[16] J. E. Shore, Minimum cross-entropy spectral analysis, IEEE Transactions on Acoustics Speech and Signal processing ASSP-29 (1981) 230-237.

[17] J. D. Markel, A. H. Gray, Linear Prediction of Speech, Springer-Verlag, New York, 1976.

[18] C. Tsallis, R. S. Mendes, A. R. Plastino, The role of constraints within generalized nonextensive statistics, Physica A 261 (1998) 534-554.

[19] A. Plastino, A. R. Plastino, From Gibbs microcanonical ensemble to Tsallis generalized canonical distribution, Phys. Lett. A 193 (1994) 140.

[20] L. Borland, A. R. Plastino, C. Tsallis, Information gain within nonextensive thermostatistics, Journal of Mathematical Physics 39 (12) (1998) 6490-6501.

[21] S. Furuichi, K. Yanagi, K. Kuriyama, Fundamental properties of Tsallis relative entropy, Journal of Mathematical Physics 45 (2004) 4868-4877.

[22] A. Hobson, A new theorem of information theory, J. Stat. Phys. 1 (1969) 383391.

[23] S. Furuichi, A characterization of the Tsallis relaitve entropy by the generalized properties, arXiv:cond-mat/0410270.

[24] E. M. F. Curado, C. Tsallis, Generalized statistical mechanics: connections with thermodynamics, J. Phys. A: Math. Gen. 24 (1991) 69-72.

[25] C. Tsallis, What are the numbers that experiments provide?, Quimica Nova 17 (1994) 468.

[26] T. Yamano, Some properties of $q$-logarithm and $q$-exponential functions in Tsallis statistics, Physica A 305 (2002) 486-496.

[27] L. Nivanen, A. L. Méhauté, Q. A. Wang, Generalized algebra within a nonextensive statistics, Rep. Math. Phys. 52 (2003) 437-434.

[28] E. P. Borges, A possible deformed algebra and calculus inspired in nonextensive thermostatistics, Physica A 340 (2004) 95-101. 
[29] H. Suyari, M. Tsukada, Law of error in Tsallis statistics, IEEE Transactions on Information Theory 51 (2) (2005) 753-757.

[30] A. Plastino, A. R. Plastino, On the universality of thermodynamics' Legendre transform structure, Phys. Lett. A 226 (1997) 257-263.

[31] R. S. Mendes, Some general relations in arbitrary thermostatistics, Physica A 242 (1997) 299-308.

[32] L. Geronimus, Orthogonal Polynomials, Consultants Bureau, New York, 1961.

[33] S. Furuichi, Chain rules and subadditivities for Tsallis entropies, arXiv:condmat/0405600.

[34] S. Martínez, F. Nicolás, F. Pennini, A. Plastino, Tsallis' entropy maximization procedure revisited, Physica A 286 (2000) 489-502. 